\title{
A study on $\Sigma-\Delta$ directional antenna array for EM energy harvesting
}

\author{
Nobuyasu Takemura ${ }^{1, \text { a) }}$ \\ ${ }^{1}$ Faculty of Fundamental Engineering, Nippon Institute of Technology, \\ Miyashiro, Minami-saitama, Saitama 345-8501, Japan \\ a)takemura.nobuyasu@nit.ac.jp
}

Abstract: A $\Sigma-\Delta$ directional antenna array for electromagnetic (EM) energy harvesting is proposed in this paper. By applying the $\Sigma-\Delta$ directional antenna array with the 180-degree hybrid circuit, it is possible to convert more electric power from the EM wave. The results of the consideration by the indoor environment using the ray tracing method are reported that the received electric power by the $\Sigma-\Delta$ directional antenna array is improved more than the single directivity antenna.

Keywords: EM energy harvesting, antenna array, hybrid circuit Classification: Antennas and Propagation

\section{References}

[1] J.A. Hagerty, F.B. Helmbrecht, W.H. McCalpin, R. Zane, and Z.B. Popovic, "Recycling ambient microwave energy with broard-band rectenna arrays," IEEE Trans. Microw. Theory Techn., vol. 52, no. 3, pp. 1014-1024, March 2004. DOI: 10.1109/tmtt.2004.823585

[2] S. Kitazawa, H. Ban, and K. Kobayashi, "Energy harvesting from ambient RF sources," Proc. 2012 IEEE MTT-S Int. Microw. Workshop Series on Innovative Wireless Power Transmission: Technologies, Systems, and Applications, THUB-2, pp. 39-42, Kyoto, Japan, May 2012. DOI: 10.1109/imws.2012.6215815

[3] H. Nishimoto, Y. Li, Y. Kawahara, and T. Asami, "A prototype implementation of wireless sensor network using RF energy harvesting," 2010 IEICE General Conference, B-20-59, p. 643, March 2010 (in Japanese).

[4] A. Sample and J.R. Smith, "Experimantal results with two wireless power transfer systems,” Proc. 2009 IEEE Radio and Wireless Symposium, MO2A-5, pp. 1618, Jan. 2009. DOI: 10.1109/rws.2009.4957273

[5] S. Kitazawa, H. Kamoda, H. Ban, N. Kukutsu, and K. Kobayashi, "Energy harvesting from broadcasting and cellular radio sources," IEICE Technical Recport, WPT2013-16, pp. 49-54, Nov. 2013 (in Japanese).

[6] K. Gudan, S. Chemishkian, J.J. Hull, M.S. Reynolds, and S. Thomas, "Feasibility of wireless sensors using ambient $2.4 \mathrm{GHz}$ RF energy," Proc. 11th IEEE International Conference on Sensor (SENSORS) 2012, pp. 1-4, Taiwan, Oct. 2012. DOI: $10.1109 /$ icsens.2012.6411176

[7] N. Takemura, "A study on $\Sigma-\Delta$ directional antenna array for electromagnetic wave energy harvesting," International Symposium on Antenna and Propagation 
(ISAP) 2020, 3C1.4, pp. 341-342, Jan. 2021. DOI: 10.23919/isap47053.2021. 9391309

\section{Introduction}

Energy harvesting technologies which convert a small amount of energy from the environment into electric power have attracted attention as a future energy source. The energy from environment sources such as light energy, thermal energy, and kinetic energy are known as ambient energy. As one of their energy sources, the electromagnetic (EM) wave is also considered because various radio waves such as wireless LAN, mobile phones, and broadcasting can be used [1, 2, 3]. It is considered to use as a recycled energy source to operate low power consumption equipment by harvesting the electric power from these EM waves. Although power harvested from electromagnetic waves is not as high as power generated by solar energy, EM wave energy is less influenced by weather and is a stable source of recycled energy. In addition, since power consumption of microcomputers and communication modules tends to reduce with each new generation, these devices can operate using harvested electric power of $\mu \mathrm{W}$ order [4]. Harvesting power of $60 \mu \mathrm{W}$ order has been reported using an antenna at a point four km away from a TV broadcasting transmission tower [5]. The radio wave harvester that convert power from communication and broadcasting radio waves has been reported [6]. The author has reported $\Sigma$ - $\Delta$ directional antenna array for EM energy harvesting to obtain more recycled energy [7].

This paper reports the statistical analysis results of the method that uses the $\Sigma-\Delta$ directional antenna array for EM energy harvesting. This antenna array is obtained the received electric power from the both $\Sigma-\Delta$ radiation patterns. The recycled energy is evaluated by the indoor environment.

\section{Antenna array structure}

Figure 1 shows the proposed $\Sigma-\Delta$ directional antenna array configuration and the image of radiation patterns. This antenna array consists of 12-element microstrip patch antenna with 180-degree hybrid circuits, a $\Sigma$-port for synthesizing signals in phase, and a $\Delta$-port for synthesizing signals in reverse phase. The output signals from the $\Sigma$ and $\Delta$ ports are commutated by the rectifier circuits and are combined to DC power by the DC combining circuit. Figures 1 (b) and (c) show radiation images for a conventional element antenna and for a $\Sigma$ - $\Delta$ directional antenna. In the case of the $\Sigma-\Delta$ directional antenna, the electric power received by the single directivity is obtained from the $\Sigma$-port, and the electric power received by the monopole directivity is obtained from the $\Delta$-port. Therefore, the electric power can be obtained at a wider angle than with the conventional antenna by receiving from both radiation patterns of the mono-directivity and the bi-directivity.

The structure and characteristics of the $\Sigma-\Delta$ directional microstrip patch antenna array is shown in Fig. 2. The $\Sigma-\Delta$ directional antenna array is formed on the dielectric substrate $(\varepsilon \mathrm{r}=2.2, \tan \delta=0.0006)$, the size is $248 \times 186 \mathrm{~mm}^{2}$, and the thickness is 


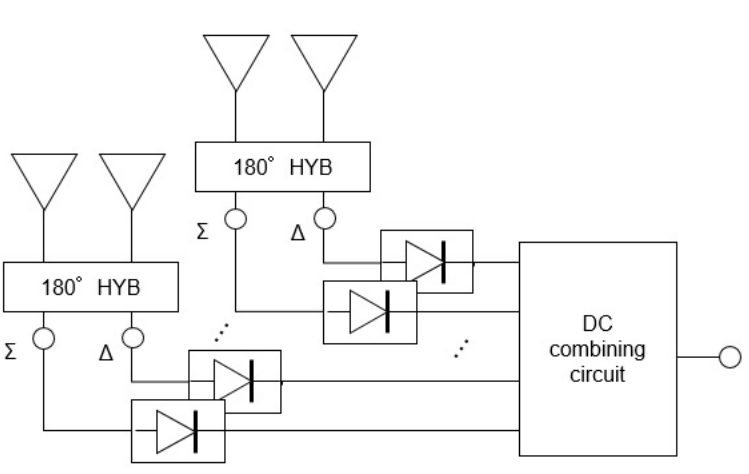

(a)

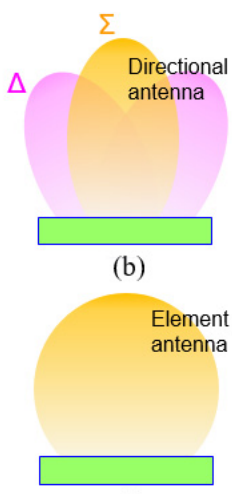

(c)

Fig. 1. Configuration of proposed antenna array and image of radiation patterns. (a) $\Sigma-\Delta$ dual-directional antenna array. (b) Radiation image of proposed directional antenna. (c) Radiation image of conventional element antenna.

$0.8 \mathrm{~mm}$. The antenna element spacing is $0.5 \lambda$ ( $\lambda$ is the wave length of the frequency $2.4 \mathrm{GHz}$ ). The antenna is calculated using Keysight EMPro software. Figures 2 (c) and (d) show the reflection and transmission characteristics of the proposed antenna. From the calculation results, the reflection characteristic is about $-14 \mathrm{~dB}$ (voltage standing wave ratio is 1.5 ) at the frequency of $2.4 \mathrm{GHz}$, and the mutual coupling of each antenna element is less than $-21 \mathrm{~dB}$. The phase characteristic of the 180-degree hybrid circuit is shown in Figs. 2 (e) and (f). In the $\Sigma$-port, the phase difference between antenna ports is 1.7 degrees and the in-phase characteristic is obtained. In the $\Delta$-port, the phase difference between antenna ports is 181.7 degrees and the reverse-phase characteristic is obtained. From the calculated radiation patterns of the proposed antenna array, the antenna array has the $\Sigma$ radiation pattern and $\Delta$ radiation pattern from the calculation results.

\section{Simulation model and results}

The recycled electric power obtained by using the $\Sigma-\Delta$ directional antenna array was calculated in an indoor environment. Figure 3 shows the simulation model and calculation results of the indoor environment for the radio propagation. The ceiling and floor of the classroom were made of concrete, the windowpanes were arranged on two sides, and the other two sides were metallic walls. Several desks made of steel were placed in the classroom. The transmitting antenna is the half-wavelength dipole imitated a wireless LAN router on the ceiling and the receiving antenna is the $\Sigma-\Delta$ directional antenna array is arranged on the desk. The transmitting dipole antenna was vertically polarized.

The radio propagation from the transmitting dipole antenna to the receiving $\Sigma-\Delta$ directional antenna array in the classroom was calculated by the ray tracing method. The calculated radiation pattern of the $\Sigma-\Delta$ directional microstrip patch antenna array shown in previous section was used in the ray tracing simulation. The received power of the $\Sigma-\Delta$ directional antenna array was calculated by the sum of the electric fields of each radio path. The received power obtained from the $\Sigma$ and $\Delta$ ports of the 


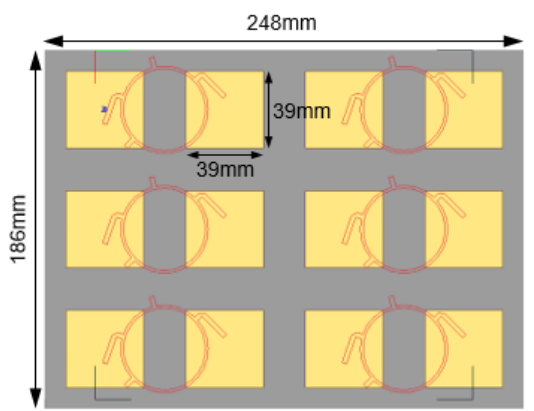

(a)

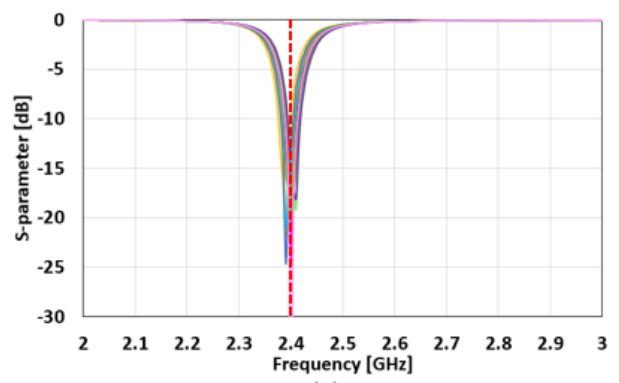

(c)

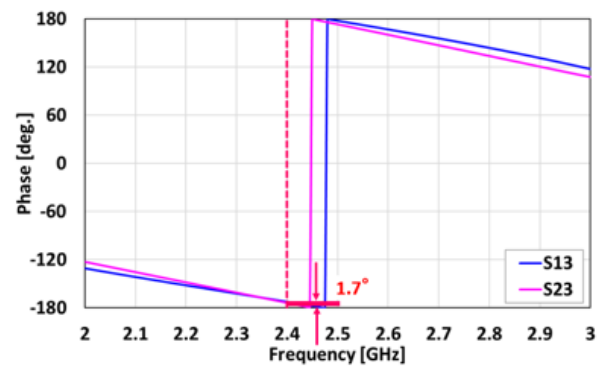

(e)

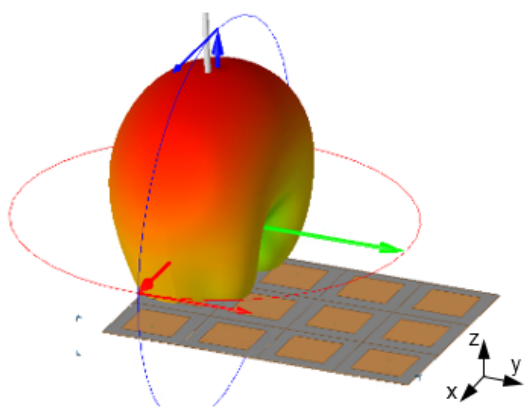

(g)

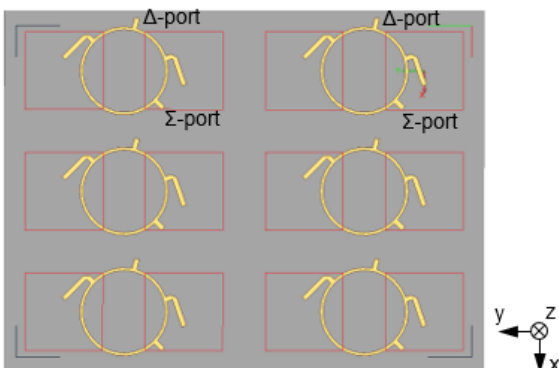

(b)

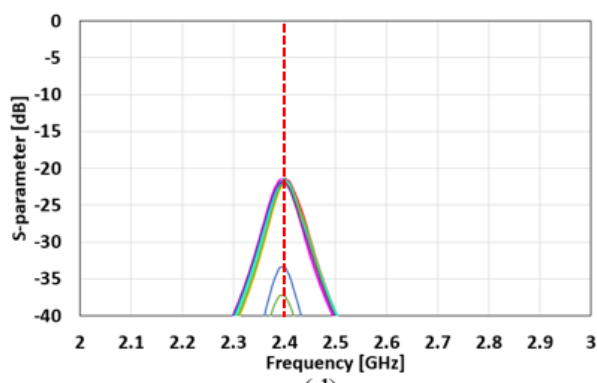

(d)

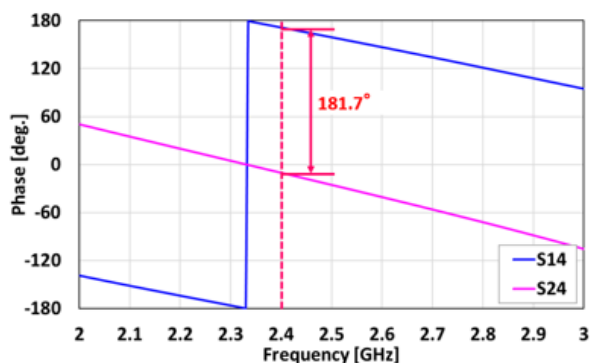

(f)

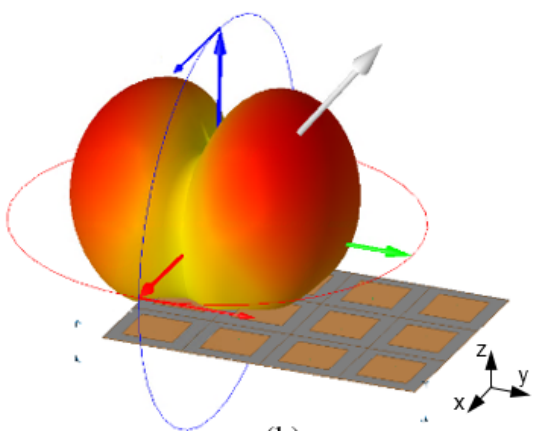

(h)

Fig. 2. Antenna array structure and calculation result of proposed antenna. (a) Front-side of antenna. (b) Back-side of antenna. (c) Reflection characteristic. (d) Transmission characteristic. (e) Phase characteristic (in-phase). (f) Phase characteristic (reverse-phase). (g) Radiation pattern $(\Sigma$-pattern). (h) Radiation pattern $(\Delta$-pattern).

antenna is as follows:

$$
\begin{aligned}
P_{R_{\_} \text {sum }} & =\left|\boldsymbol{E}_{1 \_ \text {sum }}\right|^{2}+\left|\boldsymbol{E}_{2 \_ \text {sum }}\right|^{2}+\cdots+\left|\boldsymbol{E}_{i \_ \text {sum }}\right|^{2} \\
P_{R_{\_} \text {delta }} & =\left|\boldsymbol{E}_{1 \_ \text {delta }}\right|^{2}+\left|\boldsymbol{E}_{2 \_ \text {delta }}\right|^{2}+\cdots+\left|\boldsymbol{E}_{i \_ \text {delta }}\right|^{2}
\end{aligned}
$$

where $\boldsymbol{E}_{i_{-} \text {sum }}$ and $\boldsymbol{E}_{i \_ \text {delta }}$ are the received electric fields obtained from the $\Sigma$ and 
$\Delta$ ports of the antenna. The $i$ is number of radio paths of the antenna. Therefore, the received power of the $\Sigma$ - $\Delta$ directional antenna with 180 -degree hybrid circuit is as follows:

$$
P_{R}=\frac{1}{2}\left(P_{R_{-} \text {sum }}+R_{R_{-} \text {delta }}\right)
$$

The received power was calculated for the $\Sigma-\Delta$ directional antenna array rotated 360 degrees every 10 degrees in the horizontal direction. The calculation results were evaluated by the cumulative distribution function (CDF) of the received power in the degree of each rotation angle.

The simulation conditions are shown in Table I. The received power was evaluated at 24 points in the simulation model. The conventional antenna was a 12-element microstrip patch antenna array. Figure 3 (b) shows the radio paths from the transmitting antenna to the receiving antenna at Rx position 4. Figure 3 (c) shows the $\mathrm{CDF}$ of the received power at Rx position 4 . The received power is compared the

Table I. Simulation conditions.

\begin{tabular}{l|l}
\hline Antenna position (Tx) & $3 \mathrm{~m}$ (height) \\
\hline Antenna position (Rx) & $1 \mathrm{~m}$ (on the desk, 24 points) \\
\hline Transmitting power & $10 \mathrm{~mW}$ \\
\hline Number of reflections and diffractions & 4 times and 2 times \\
\hline Angular resolution of ray & 1 degree \\
\hline
\end{tabular}

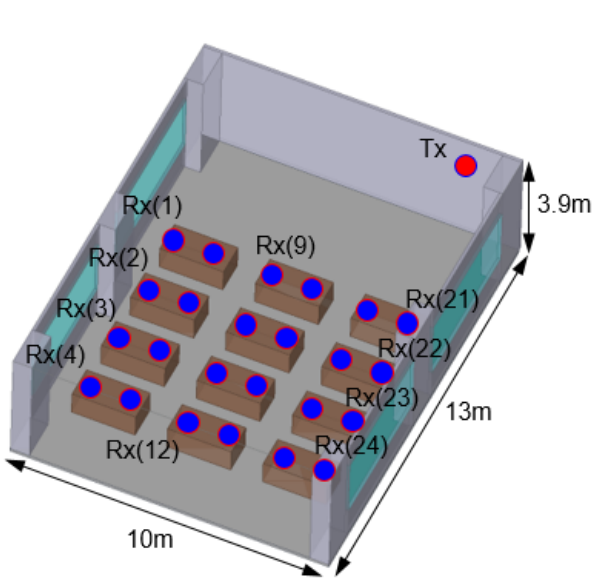

(a)

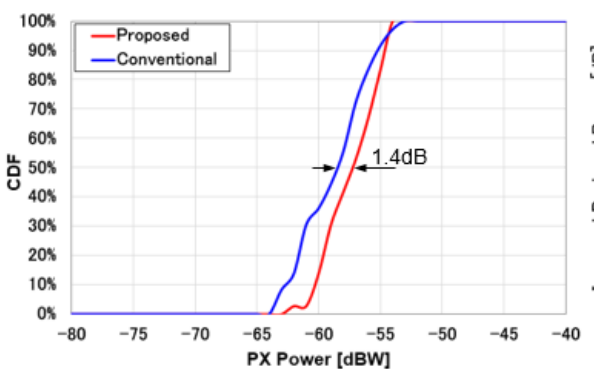

(c)

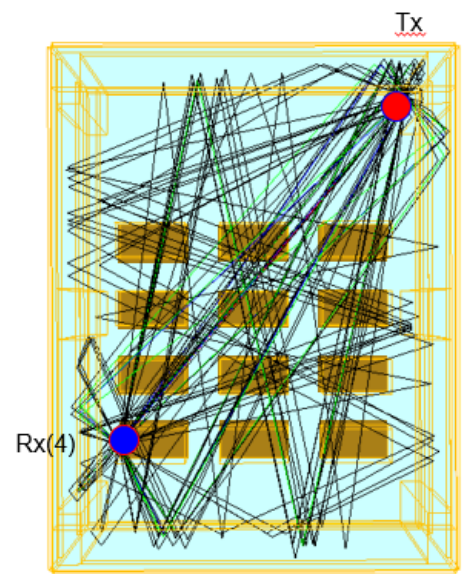

(b)

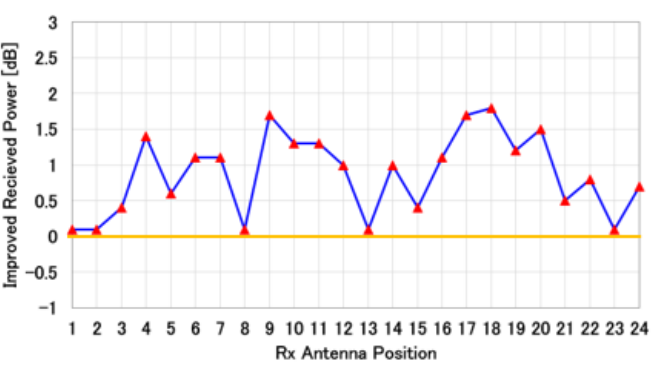

(d)

Fig. 3. Simulation model and calculation result of radio propagation. (a) Simulation model of class room. (b) Radio paths from transmitting to receiving antenna. (c) CDF at $\mathrm{Rx}$ position 4. (d) Improved received power at each $\mathrm{Rx}$ antenna position. 
conventional microstrip patch antenna array with single directivity. The $50 \%$ value of the CDF was $-58.8 \mathrm{dBW}$ and $-57.4 \mathrm{dBW}$ for the conventional antenna and the proposed antenna, respectively. The received power has improved by $1.4 \mathrm{~dB}$. Figure 3 (d) shows the improved received power compared to the conventional antenna array. From the simulation results, the received power improved by using the $\Sigma$ - $\Delta$ directional antenna array at the all received point.

\section{Conclusion}

The $\Sigma-\Delta$ directional antenna array for EM energy harvesting has been proposed as a method for improving the received electric power from EM wave. The proposed antenna array was realized by using the 12-element microstrip patch antenna with 180-degree hybrid circuits. The received electric power has been calculated by the indoor environment of classroom using the ray tracing method. The received power was calculated for the proposed antenna array rotated 360 degrees in the horizontal direction, and was evaluated by CDF of the received power in the degree of each rotation angle. It was confirmed that the recycled energy was improved by using the $\Sigma-\Delta$ directional antenna array.

\section{Acknowledgments}

This research was partially supported by Research Grant for Exploratory Research of Power Academy. 